\title{
Escherichia coli Growth Dynamics: A Three-Pool Biochemically Based Description
}

\author{
Abhay Joshi and Bernhard O. Palsson \\ Department of Chemical Engineering, The University of Michigan, \\ Ann Arbor, Michigan 48109-2136
}

Accepted for publication November 26, 1986

\begin{abstract}
A three-pool growth model of an individual Escherichia coli cell is described herein. The model is based on a previously developed chemically structured complex single cell growth model. The reduction in model complexity and the identification of the essential modes of motion, over the time scale of growth, is achieved by temporal decomposition and analysis of hierarchy in relaxation times. The three-pool model faithfully simulates the changes in cell size, cell shape, cell macromolecular composition, DNA initiation and termination periods, and the dependence of cell growth under abiotic glucose limitation. The predictions made by the reduced model compare favorably with both the experimental data and those of the full single cell model (SCM) without any parameter adjustments. The three-pool model has very few significant parameters and has the potential to find immediate practical use in bioreactor design and process control strategies. The model development illustrates the use of modal analysis to yield reduced physiologically realistic dynamic model of complex microbial system such as E. coli.
\end{abstract}

\section{INTRODUCTION}

A bacterial cell is a self-contained biochemical reactor which has the capacity to catalyze and regulate a series of complex reactions. The prokaryotic cell consists of structured aggregates of macromolecules which in turn are made from the pools of precursors of lower molecular weight. The precursor pools are derivatives of glucose, or some other carbon source, and they are continuously replenished by biochemical syntheses. Accordingly, cellular growth is an incessant dynamic activity of more than 2000-3000 individual chemical reactions. ${ }^{1}$ These chemical reactions can be broadly categorized as biosynthetic, polymerization, and fueling reactions.

The idea of describing such a complex cellular activity with a mathematical model is not new to researchers. Considerable theoretical work on modeling the growth of cells has already been carried out. A simple functional relationship between the specific growth rate and the substrate concentration was proposed by Monod in 1942 as being a hyperbolic Michaelis-Menten type of expression. Since the pioneering work of Monod, many researchers have tried to explain the growth dynamics of different kinds of cells with varying degree of modeling constraints and limitations. Pollard and Yeisley ${ }^{2,3}$.described the synthetic processes of a bacterial cell using 5 and 7 differential equations. More rigorous attempt was made by Heinmets ${ }^{4}$ who modeled the cell using 19 differential equations to account for various transients processes. The model had the limitation of continuous growth with arbitrary imposition of the criteria of cell division. Weinberg and Ziegler ${ }^{5}$ described the transient behavioral pattern of the nondividing cell. Davison ${ }^{6}$ modeled a dividing cell with 17 differential equation with no explicit accounting for the abiotic environment. All these models were either phenomenological or had limited accounting of the biochemical and physiological events during the cell growth.

The major metabolic pathways are now well documented and they have been characterized in terms of stoichiometry and biochemical kinetics. This opens the possibility for developing physiologically realistic growth models which account for well documented biochemical events. Such modeling would incorporate some biochemical structure to explain the dynamic behavior of the cell. Various metabolic models have been proposed to simulate the environment host-cell interaction based on the underlying chemical structure. ${ }^{7-15}$ Researchers have also proposed genetically structured models that accounts for detailed structure and function of relatively small genomes as plasmids. ${ }^{16-18}$ It is evident that the introduction of the detailed chemical structure will result in an enormously complex mathematical description.

A comprehensive chemically structured model for the bacterium Escherichia coli has been formulated by Shuler and colleagues ${ }^{11-14}$ to describe growth under either carbon or nitrogen source limitation. The growth model under glucose limitation consists of a detailed description of $18 \mathrm{key}$ metabolites and the criteria for DNA initiation, termination, and cell division and it contains over a 100 parameters which can be obtained from the literature or from objective heuristic rules. This detailed but still lumped single cell model (SCM) has made a noteworthy contribution to the 
understanding of the cellular growth processes but may be too complex for routine practical applications, such as for efficient bioreactor design and direct on-line process control strategies. A balance between physiological reality and simplicity calls for reduction in the complexity of the mathematical description to develop a tractable model that is of practical utility.

The SCM serves as a starting point for the development of a simplified but physiologically rational $E$. coli growth model. The important task is to extract the dynamic essentials from the complex model that are necessary to describe the growth process itself. Conceptualization and model reduction are two major difficulties encountered in the study of organizationally complex system such as the SCM. ${ }^{19,20}$ We will show how both these difficulties can be overcome by a "modal decomposition" technique which has recently been shown to be useful to interpret biochemical kinetics. ${ }^{21-23}$ This approach is based on the analysis of internal mechanism of the system and the recognization of the existence of multiple time scales in the metabolic network.

We will demonstrate that modal decomposition naturally and logically reduces the dynamic order of the complex glucose-limited SCM to yield results that are consistent with physiological function. A three pool model description of $E$. coli growth is developed which represents a compromise between the oversimplified Monod model and the SCM. The reduced modelcompares favorably with the full SCM and growth data.

\section{TEMPORAL DECOMPOSITION OF DYNAMIC MODELS}

\section{Importance of Intrinsic Time Scales for Model Simplification}

Each individual biochemical event (a chemical reaction, a transport process, etc.) that participates in the growth process proceeds with a characteristic time scale and contributes to the overall cellular response. Through interactions of the individual events, the integrated system exhibits motion on several time scales. These time constants are systemic properties and they are usually distributed over several orders of magnitudes. ${ }^{19,26}$

From a practical point of view, one is interested in the metabolic response that overlaps with external process or observational time scales. ${ }^{19,20,26}$ Owing to the large dimensionality of the interconnected metabolic mesh, it is uneconomical and perhaps even impossible to represent the entire system model in detail. A large number of approaches have been used to resolve the problem of reducing the dimensions of the complex systems. This includes the heuristic method of lumping the parameters together, compartmentalizing various processes into one, representing a priori a series of first order systems as one black box, etc.

The main challenge in analyzing such systems is to assess both the overall time constants and the dynamic interaction between the system variables. Modal analysis can provide this information. ${ }^{19-23}$

\section{Modal Decomposition}

Behavioral patterns of biological systems are intrinsically nonlinear. Linear approximation, however, can give qualitative information about the properties of nonlinear kinetic equations. The difficulty of analyzing the nonlinear dynamic characteristics can therefore be partially resolved by linearizing the set of kinetic equations at the expense of mathematical rigor. Linearization of the dynamic equation is achieved by expanding them into a Taylor series around a reference state as:

$$
\frac{d}{d t}(\mathbf{x})=f(\mathbf{x})=f\left(\mathbf{x}_{\text {ref }}\right)+\mathbf{J}\left(\mathbf{x}-\mathbf{x}_{\text {ref }}\right)+\ldots
$$

where $\mathrm{x}$ is an $n \times 1$ vector of metabolite concentrations; $f(\mathbf{x})$ is an $n$-dimensional nonlinear function which contains the reaction rate laws; and $\mathbf{J}$ is the Jacobian matrix of order $n \times n$. The subscript 'ref' denotes the reference conditions. Each entry in $\mathbf{J}$ is determined by evaluating $\left(d f_{i} / d x_{j}\right)$ at the reference condition. The reference points may be the initial states, stationary states or any point along the nominal trajectory. The following information is obtained through linear analysis.

\section{The Time Constants or the Relaxation Times}

The characteristic equation, $\operatorname{det}(\mathbf{J}-\boldsymbol{\lambda} \mathbf{I})=0$, is solved for the eigenvalues $\lambda_{1}, \lambda_{2}, \lambda_{3}, \ldots, \lambda_{n}$. The negative reciprocals of the eigenvalues are the time constants. Evaluation of the time constants gives the distribution of time scales inherent in the system.

The hierarchy of intrinsic intracellular times can be represented by time axis, ${ }^{20,26}$ in Figure 1. Fast transients are characterized by the processes at the extreme left and slow transients at the extreme right. The process time scale, i.e., the time scale of interest can be represented by a window of observation on this time axis. The mechanisms which have the time constants faster than the observed window can be eliminated from the dynamic description as these mechanisms have reached a quasi steady state. However, the mechanisms which have the transients slower than the observed time exhibit high "inertia" and hardly move from their initial state. Hence, the fast and slow transients, as compared to the transients of the observed window of time scales, can be removed from the dynamic description of the system. However, the difficult task of elucidating how the metabolite concentrations move on these time scales still remains.

\section{The Dynamically Independent Modes}

The Jacobian matrix obtained from linearization can be diagonalized (there are some restrictions on this process, but are not of concern here) by the transformation $\mathbf{J}=\mathbf{M} \mathbf{\Lambda} \mathbf{M}^{-1}$ where the matrix $\mathbf{\Lambda}$ has the eigenvalues of $\mathbf{J}$ on the diagonal and $\mathbf{M}^{-1}$ and $\mathbf{M}$ are constant matrices comprised of the eigenrows and the eigenvectors respectively. A new realization $\mathbf{m}$ can be expressed as a linear combination of the state 


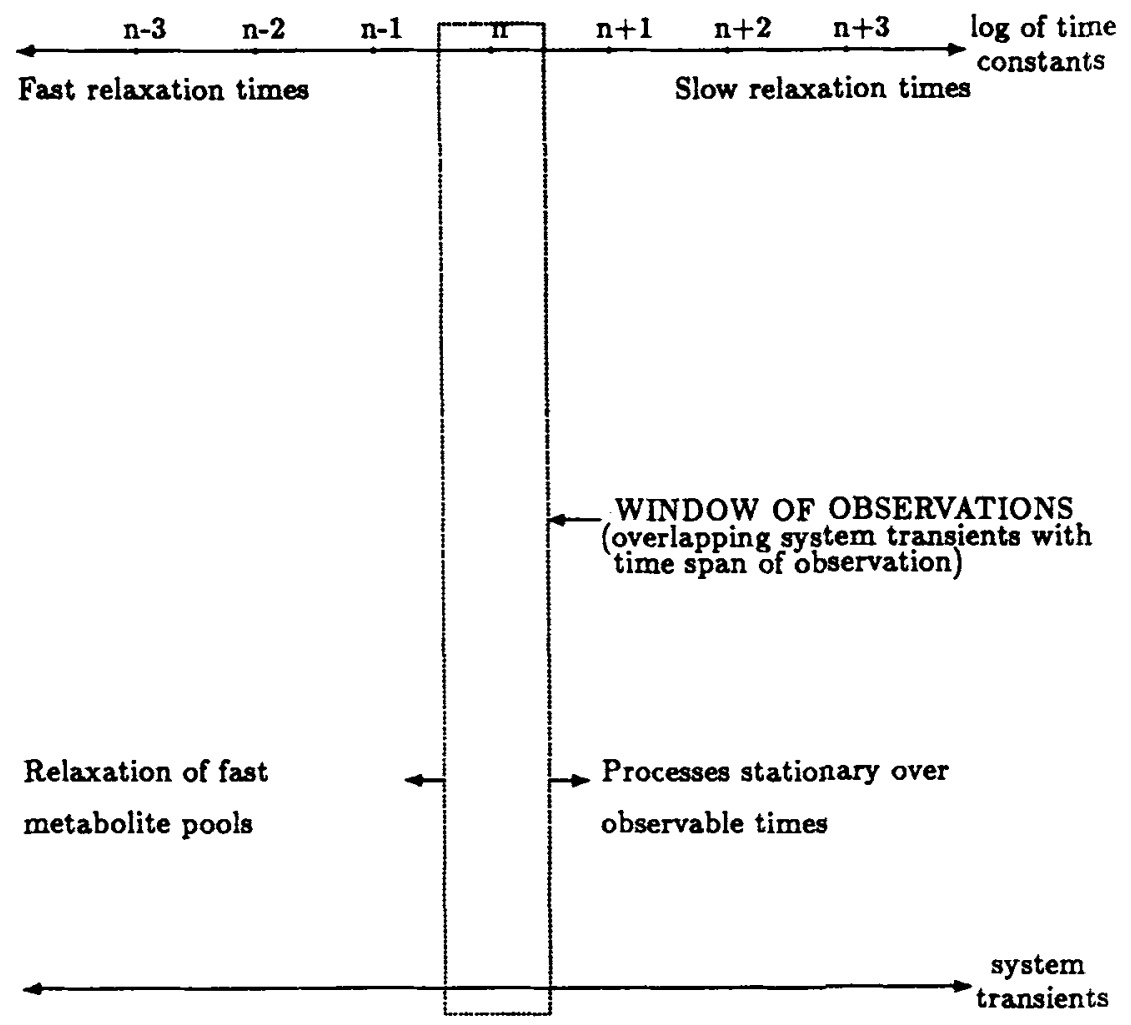

Figure 1. Schematic illustration of system transients that overlap with the time span of observation; $n, n+1, \ldots$ represent the decadic order of time constants.

variables $\mathbf{x}$ as $\mathbf{m}=\mathbf{M}^{-1} \mathbf{x}$. The linearized system is transformed into a set of dynamically independent variables as

$$
\frac{d \mathbf{m}}{d t}=\Lambda \mathbf{m}+\mathbf{M}^{-1}\left[f\left(\mathbf{x}_{\text {ref }}\right)-\mathbf{J} \mathbf{X}_{\text {ref }}\right]
$$

or

$$
\frac{d \mathbf{m}}{d t}=\Lambda \mathbf{m}+\mathbf{K} ; \quad \mathbf{K}=\text { const } .
$$

The modal matrix $\mathbf{M}^{-1}$ is defined by

$$
\mathbf{M}^{-1}=\left(\begin{array}{cccc}
u_{11} & u_{12} & \cdots & u_{1 n} \\
u_{21} & u_{22} & \cdots & u_{2 n} \\
\cdot & \cdot & \cdots & \cdot \\
u_{n 1} & u_{n 2} & \cdots & u_{n n}
\end{array}\right)=\left(\begin{array}{c}
\mathbf{u}_{1} \\
\mathbf{u}_{2} \\
\cdot \\
\mathbf{u}_{n}
\end{array}\right)
$$

where $\mathbf{u}_{1}, \mathbf{u}_{2}, \mathbf{u}_{3}, \ldots, \mathbf{u}_{n}$ are the eigenrows of the system. The eigenrows are found by solving $\mathbf{u}_{i} \cdot\left(\mathbf{J}-\lambda_{i} \mathbf{I}\right)=0$. The new variables $\mathbf{m}$ are called modes. They can be expressed as:

$$
\begin{gathered}
\mathbf{m}=\mathbf{M}^{-1} \mathbf{x} \\
\Rightarrow m_{i}=\mathbf{u}_{i} \cdot \mathbf{x}=\sum_{j=1}^{n} u_{i j} x_{j} \\
=u_{i 1} x_{1}+u_{i 2} x_{2}+u_{i 3} x_{3}+\ldots+u_{i n} x_{n}
\end{gathered}
$$

where $u_{i j}$ is the measure of the contribution of variable $j$ in mode $i$. For instance, if an eigenrow has a dominant element, say $u_{i j}$, then $m_{i} \simeq x_{j}$. Under such conditions, $x_{j}$ is a decoupled dynamic variable and moves on a time scale given by $\lambda_{i}$.

The modal matrix $\mathbf{M}^{-1}$, therefore, maps the concentration variables onto the timescales and it tells us how various metabolites move on a particular time scale. The eigenvectors are only specified with a multiplicative constant and can therefore can be scaled to our convenience.

Equation (3) can be integrated with $\mathbf{m}(t=0)=\mathbf{m}_{0}$ to give

$$
\begin{aligned}
\mathbf{m}(t)=\mathbf{m}_{0} e^{\Lambda t}+\int_{0}^{\tau} \mathbf{K} e^{(t-\tau) \Lambda} d \tau & \\
& =\left(\mathbf{m}_{0}+\Lambda^{-1} \mathbf{K}\right) e^{\Lambda t}-\Lambda^{-1} \mathbf{K}
\end{aligned}
$$

or

$$
m_{i}(t)=a e^{\lambda_{i} t}+b
$$

where $a$ and $b$ are appropriately defined constants.

Equation (6) shows that the modes move independently of each other on timescales defined by the corresponding eigenvalue. We can therefore think of the linear system specified by the representation $\mathbf{m}$ as a set of $n$ decoupled first order subsystems, i.e., each subsystem evolves in time independently of the others. This decomposition is important when it is desired to focus attention on some important subset of the modes, e.g., the fastest or the slowest ones. In such case we can reduce the dimension of the problem by ignoring the dynamics of the other modes. ${ }^{19-23}$ 
THE STRUCTURED SINGLE-CELL MODEL OF ESCHERICHIA COLI

Shuler and co-workers ${ }^{11,12}$ have developed a SCM for glucose-limited growth of the bacterium $E$. coli. This model is a comprehensive description of the underlying biochemistry and physiology of cellular events. The E. coli SCM visualizes the "whole cell" as an expanding biochemical reactor free to change its size and shape and to respond to the changes in the external glucose and ammonium concentration. The SCM has the capacity to predict the changes in cell morphology and cellular composition during growth. Within the framework of defined parameters, the cell size and shape, length of $C$ and D phases, points of DNA initiation and termination, and response of the cell to changes in external glucose concentration are natural responses of the model.

\section{Model Structure and Assumptions}

The following comments are made with regard to the SCM:

1) The model formulation is based on certain simplifying assumptions: internal reactions of the cell are not diffusion limited; mineral nutrients are in excess; waste accumulation is negligible in the cell; and the cell division results in two identical daughter cells. The mechanism for the control of DNA initiation proposed by Fralick and Messer and coworkers ${ }^{27-29}$ is incorporated in the cell model. The geometry of the cell is specified by assuming the cell to be cylindrical with hemispherical ends and the cell division results at the formation of two hemispherical ends at the cell's center as cross wall. ${ }^{11-14}$
2) Using the above assumptions, the complete model for the cell growth under glucose limitation accounts for 18 metabolite concentrations which represent the inherent dynamic structure of the underlying metabolism. Each equation in this model corresponds to a dynamic mass balance on a given metabolite. The model equations are too complicated to justify a detailed listing here. Such a listing can be found in the original reference ${ }^{12}$ though a brief description of the model is given in Figure 2.

The entire model can be viewed as a dynamic intermediary metabolism that is coupled to 'auxiliary' descriptions related to cell morphology and decision making processes in the cell. The metabolism is considered to consist of three major parts: a) the catabolic reactions that provide the ATP and the reducing power needed for biosynthesis; b) the anabolic reactions that produce the building blocks needed for polymerization reactions; and c) the epigenic system that accounts for the synthesis of macromolecules-DNA, RNA, protein, and cell wall.

3) Cell morphology and certain decision making events are explicit functions of the concentrations of macromolecules present. A set of 'auxiliary equations' corresponds to the cell morphology, septum wall synthesis, and DNArelated events and are derived directly from the metabolic description. Such a topological structure of $E$. coli growth is given in Figure 2.

4) Intermediary metabolism and auxiliary description together contain over 100 parameters. Justification and independent experimental determination of these parameters is exceedingly difficult and the complexity of the model hampers clear understanding of the sensitivity of the overall growth pattern to these parameters.

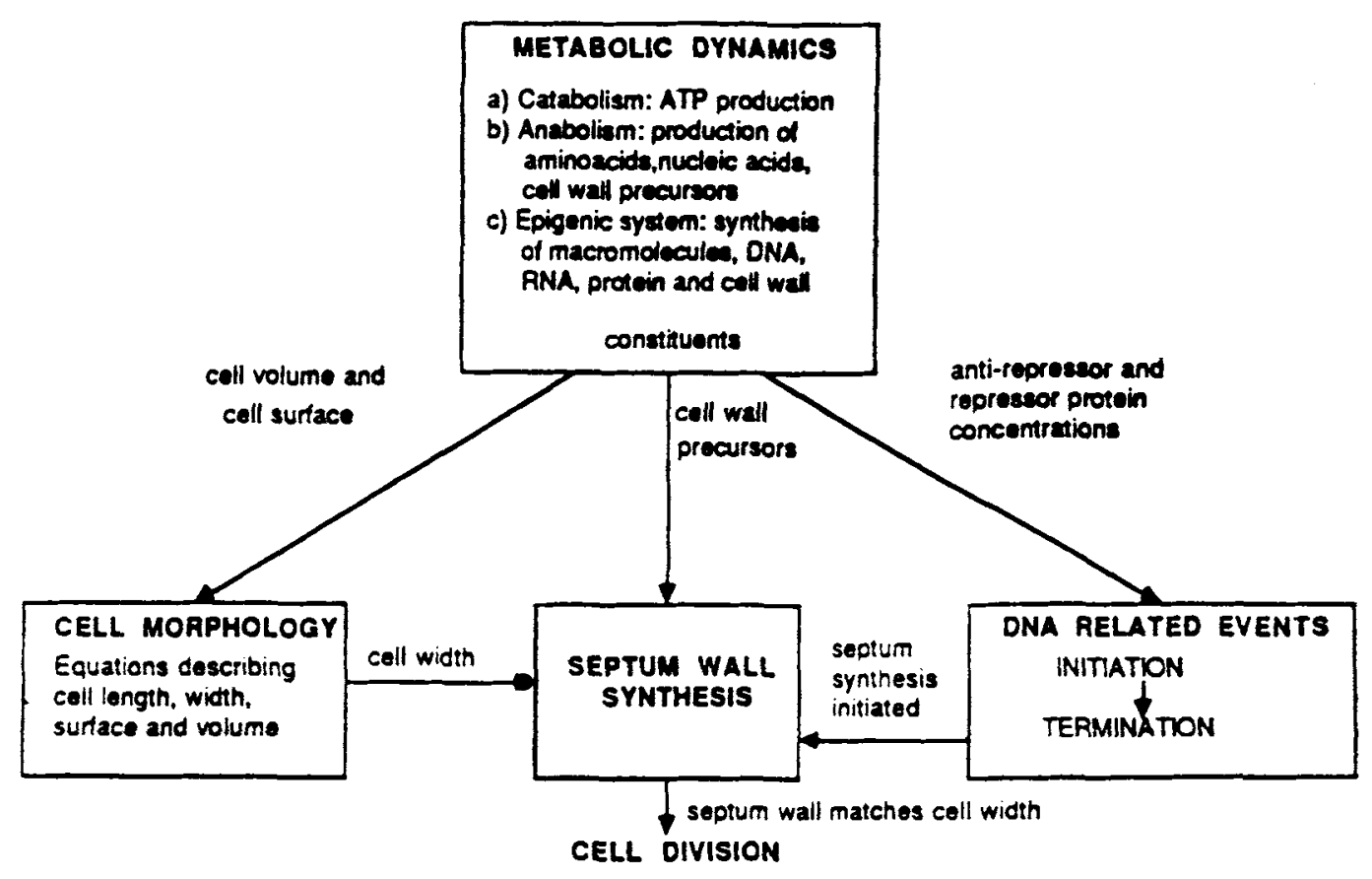

Figure 2. Topological structure of $E$. coli growth model. 


\section{Rate Laws}

Each of the equation used in the SCM represents a multiple saturation type of enzyme kinetic expression having three major factors:

a maximum velocity factor, $V_{m_{i}}$;

a saturation factor

$$
\left(x_{i} / V\right) /\left(K_{x_{i}}+x_{i} / V\right)
$$

for each reactant; and a regulatory factor

$$
K_{x_{i}} /\left(K_{x_{i}}+x_{i} / V\right)
$$

In the above expressions $V_{m_{i}}$ is the maximum velocity factor; $K_{x_{i}}$ is the saturation constant; $V$ is the volume of the cell; $x_{i}$ is the mass of the metabolite $i$ per cell; and $x_{i} / V$ is the concentration of the metabolite $i$ in the system. The rates depend on the substrate in the Michaelis-Menten fashion.

The crucial problem in a model of such complexity is the determination of stoichiometric and kinetic parameters. Justification of the kinetic parameters provided by the authors ${ }^{11-13}$ show that these parameters could be obtained directly from the literature. Most of the saturation constants were obtained from general heuristic rules governing the relationship of saturation constants to normal intracellular concentration of the affecting metabolite. Typical fractions ${ }^{11-14}$ used were $1 / 5,1 / 7,1 / 25,1 / 90,1 / 400$, etc., with the fact that the definition of the normal intracellular concentration is somewhat arbitrary.

It is clear from above that most of the metabolite concentrations are well in the saturation region of the used -Michaelis-Menten curves. Since the metabolite mass and the cell volume grow exponentially with time during the cell cycle, the metabolite concentration inside the cell do not change significantly during the growth process. As a result the kinetic expressions with the terms of the form $\left(x_{i} / V\right) /$ $\left(K_{x_{i}}+x_{i} / V\right)$ and $K_{x_{i}} /\left(K_{x_{i}}+x_{i} / V\right)$ in the SCM are almost frozen to the initial value close to unity except under the transient situations when the abiotic substrate concentration is changed (stepped up or stepped down) and the cell takes a few generations of growth to achieve a new steady state growth rate.

\section{The Dynamic Mass Balances}

As an illustration of how the SCM is formulated we show the equations from the original model that describe the formation of cell envelope precursors and cell envelope constituents:

$$
\begin{array}{r}
\frac{d P_{4}}{d t}=k_{4}\left(\frac{K_{P_{4}}}{K_{P_{4}}+P_{4} / V}\right)\left(\frac{P_{1} / V}{K_{P_{4} P_{1}}+P_{1} / V}\right) \\
\times\left(\frac{A_{2} / V}{K_{P_{4} A_{2}}+A_{2} / V}\right) V-\gamma_{4} \frac{d M_{4}}{d t}
\end{array}
$$

$$
\begin{aligned}
\frac{d M_{4}}{d t}= & \mu_{4}\left(\frac{P_{4} / V}{K_{M_{4} P_{4}}+P_{4} / V}\right)\left(\frac{A_{2} / V}{K_{M_{4} A_{2}}+A_{2} / V}\right) \\
& \times\left(E_{2}+E_{3}\right)-K_{T M_{4}}\left(\frac{A_{2} / V}{K_{T M_{4} A_{2}}+A_{2} / V}\right) M_{4}
\end{aligned}
$$

where $P_{4}$ is the amount (mass units per cell) of cell envelope precursors; $M_{4}$ is the amount of nonprotein part of the cell envelope; $P_{1}$ is the amount of amino acids; $A_{2}$ is the amount of intracellular glucose; $E_{2}$ and $E_{3}$ are the amount of enzymes involved in the septum wall formation and cell envelope synthesis; and $t$ is the time. The rate constants for the maximum rate of precursors and cell envelope synthesis are $k_{4}, \mu_{4}$, and $K_{T M_{4}} ; \gamma_{4}$ is a stoichiometric coefficient; and $K_{P_{4}}$, $K_{P_{4} P_{1}}, K_{P_{4} A_{2}}, K_{M_{4} P_{4}}, K_{M_{4} A_{2}}$ and $K_{T M_{4} A_{2}}$ are "Michaelis" and inhibition constants. Values of $K_{P_{4}}$ and $K_{P_{4} P_{1}}$ in the full SCM were estimated as a certain fraction of the normal intracellular values of $P_{4}$ and $P_{1}$.

Two obvious questions arise: how can we resolve the degree of complexity of the SCM? Which of the kinetic parameters strongly affect the growth rate under multitude of growth conditions? As we will see, in the Reduced Model Description section, the model reduction process eliminates a bulk of the kinetic parameters and concentrates them into a significant few.

\section{SCM AND REDUCTION OF METABOLIC DYNAMICS}

The metabolic part of this model is a key to the dynamic description of cellular growth. As indicated above, the dynamic description used is quite extensive but to make the model readily usable and implementable there is a considerable need to eliminate any model complexity which is not of interest for a particular modeling objective. Our attempt at model simplification is based on the comparison of intrinsic relaxation times and the time span of cellular growth as detailed previously. The objective is to develop a simple tractable model for glucose limited $E$. coli growth that accounts for macromolecule formation and satisfy glucose dissimilation requirements to meet ATP need for biosynthesis. Concomitantly, the reduced model should predict cell size, shape, growth rates and overall composition with a few well characterized set of kinetic parameters.

\section{Development of a SCM Simulator}

A computer program that simulates the full SCM model was written using the EPISODE package as the differential equation solver. ${ }^{30}$ The program has the ability to change the step size to accommodate varying degree of stiffness in the equations. All predictions from this simulated model were compared with that of the published work ${ }^{11-14}$ and were found in close agreement. The simulated model predicted the growth rates under the glucose limitations.

The program was allowed to generate steady state growth patterns for a set of initial conditions whose output was a 45-min doubling cell. This output was categorized as a set 
of initial conditions for later use. The abiotic glucose concentration was changed from the set of initial conditions and the program was allowed to run with this new external glucose concentration for several generations until the cell reached the steady state cycle of growth with a new doubling time. Thus several sets of initial condition of metabolite compositions were obtained along with their corresponding doubling times. These metabolite compositions were then used as reference conditions to initiate the modal decomposition process to reduce the complexity of the model.

\section{Comparison of Relaxation Times and Temporal Decomposition}

A careful investigation of the full model shows that only 14 equations are dynamically independent. The concentration of the metabolite ppGpp is three orders of magnitude lower than the lowest initial concentration of precursors and low levels of ppGpp are maintained during the balanced growth of the cell. ${ }^{31}$ Nevertheless, upon glucose and amino acid starvation, rapid synthesis and accumulation of ppGpp above basal level exert stringent control of RNA synthesis. Thus, the quick production of large amounts of ppGpp in response to glucose or amino acid starvation reduces the rate of synthesis of RNA, rate of synthesis of proteins and has an effect on the protein synthesizing system of the cell. This effect of ppGpp can be accounted implicitly by making the anabolic rate $\nu_{\text {ana }}$ depend on external glucose concentration. Consequently, ppGpp was eliminated from the model.

The time constants and the modal matrix of the full model for a newly born cell with a doubling time of $45 \mathrm{~min}$ was calculated using efficient computer codes, namely, EISPACK ${ }^{32}$ The time constants for a newly born $45-\mathrm{min}$ $E$. coli cell and the contribution of modes to these relaxation times is shown in Table I. The information displayed in this table reveals important dynamic interpretations of this model at the reference conditions. The inherent time constants in the system show that the metabolic transients can be divided into three time regimes.

1) Transients faster than growth (of the order of seconds to minutes): The fast transients are associated with the reactions that adjust rapidly to the growth pattern. The transients due to these mechanisms relax very quickly and play an insignificant role in the system description in the realm of observable times of cell growth. These transients represent the low-molecular-weight metabolism and their significance has been elucidated earlier. ${ }^{19,33}$

2) Transients slower than growth (of the order of days): Mechanisms corresponding to these transients exceed the characteristic observation times of the process. The slowest dynamics in the model represent a slowly changing mass balance on the septation enzyme and glycogen and they are too slow to become significant over growth times. For all practical purposes, these dynamic balances can be assumed to be frozen in their initial states and can be subsequently eliminated from the system description.

3) Growth dynamics and overlapping metabolic transients (on the order of tens of minutes to an hour): These transients are comprised of three modes indicating that the essential dynamic order of the metabolic model is three; therefore a three-equation model should be sufficient to describe the metabolic transients that overlap with the dynamics of cellular growth.

\section{Growth Dynamics and Overlapping Metabolic Transients}

The first mode in Table I has a positive eigenvalue resulting in an exponential growth pattern. The relationship between the linearized growth time constant and the doubling time of the cell is given by the relation:

$$
t_{\text {double }}=-\ln (2) t_{\text {growth }}
$$

where $t_{\text {growth }}$ is the time constant of the unstable growth mode. The specific growth rate of the cell is given by the reciprocal of $t_{\text {growth }}$. This time constant gives an excellent prediction of the cycle time of the cell (note that this prediction is based only on the initial conditions specified for the cell and no full integration of the model equations is involved $^{33}$ ).

Table I shows that the proteins and amino acids move in a constant ratio on these times and are dynamically equivalent. Similar observations are made for the pools of cell envelope precursors and its constituents and ribonucleotides, deoxyribonucleotides and RNA. These metabolic pools are defined as

Table I. Modal matrix of $E$. coli for a 45 -min. cell. The symbols used are $\left(A_{1}\right)$ ammonium ions, $\left(A_{2}\right)$ glucose, $\left(P_{1}\right)$ amino acids, $\left(P_{2}\right)$ ribonucleotides, $\left(P_{3}\right)$ deoxy ribonucleotides, $\left(P_{4}\right)$ cell envelope precursors, $\left(M_{1}\right)$ proteins, $\left(M_{4}\right)$ cell envelope constituents, $\left(M_{5}\right)$ glycogen, $\left(E_{3}\right)$ septation enzyme, $\left(\mathrm{RNA}_{1}\right.$, $\mathrm{RNA}_{2}, \mathrm{RNA}_{3}$ ) different forms of $R N A$. The matrix represents the three essential growth modes of motion. Modes $2-9$ are comprised of fast transients and are in a quasisteady state; modes 12 and 13 represents slow inertial transients. Interaction of $P_{4}$ and $M_{4}$ on the growth time scales are underlined and is discussed in the text as an example for model reduction (ref. 33).

\begin{tabular}{|c|c|c|c|c|c|c|c|c|c|c|c|c|c|c|}
\hline $\begin{array}{l}\text { Mode } \\
\text { no. }\end{array}$ & $\begin{array}{c}\text { Time } \\
\text { constants }\end{array}$ & $A_{1}$ & $A_{2}$ & $P_{1}$ & $P_{2}$ & $P_{3}$ & $P_{4}$ & $M_{1}$ & $M_{4}$ & $M_{5}$ & $E_{3}$ & $\mathrm{RNA}_{1}$ & $\mathrm{RNA}_{2}$ & RNA \\
\hline 1 & $-0.95 h$ & 0.5 & 0 & 0.1 & -0.1 & -0.1 & $I$ & 0.1 & 1.1 & 0 & 0.7 & -0.1 & -0.1 & -0.1 \\
\hline 2-9 & $<\min$ & & & & & & & & & & & & & \\
\hline 10$\}$ & $\mathrm{h}$ & 0.2 & 0 & 0.1 & 0.5 & 0.5 & -1 & 0.1 & -1.1 & -0.1 & 0 & 0.6 & 0.6 & 0.5 \\
\hline 11$\}$ & & 0.5 & 0 & 0.3 & -0.5 & -0.5 & 1 & 0.5 & 1.1 & 0 & 2 & -0.5 & -0.5 & -0.5 \\
\hline $12-13$ & $>10 h$ & & & & & & & & & & & & & \\
\hline
\end{tabular}


pool $_{1}$ - ribonucleotides, deoxyribonucleotides, and RNA; pool $_{2}$ - amino acid and protein; and

pool $_{3}$-Cell envelope precursors and cell envelope constituents.

This implies that on the order of tens of minutes to hours, these three key metabolite pools represent the essential behavior of $E$. coli metabolism during growth. Similar observations are made when the modal matrices for the $E$. coli cells with cycling times of $0.84,1.34,2.18$, and $2.78 \mathrm{~h}$ are calculated. One important point to note here is that the relative contribution of the metabolites to the pools is constant for all the cycle times though the relative contribution of pools to the modes may vary. Since we use the pools rather than the modes as our dynamic variables, the conclusions drawn from the modal matrix becomes independent of the reference states; clearly an important result.

\section{REDUCED MODEL DESCRIPTION}

The development of the reduced dynamic growth description is presented in two steps. We first present the use of the information obtained through linear analysis.

\section{Linear Formalism - The Steady Growth Model}

\section{Modal Reduction}

We will now use the coefficients in the modal matrix to combine the original differential equations into three equations as prescribed by the linear analysis. To avoid too much detail we continue to use the dynamic mass balances on cell envelope precursors and constituents for illustration. Equations (6) and (7) can be written in the most simplified form as:

$$
\begin{gathered}
\frac{d P_{4}}{d t}=\alpha_{P_{4}} V-\gamma_{4} \frac{d M_{4}}{d t} \\
\frac{d M_{4}}{d t}=\alpha_{M_{4}}\left(E_{2}+E_{3}\right)-\gamma_{M_{4}} M_{4}
\end{gathered}
$$

The factors $\alpha_{P_{4}}, \alpha_{M_{4}}$, and $\gamma_{M_{4}}$ can be viewed as macroscopic constants obtained by combining the first-order reaction rate constants with the maximum saturation rate. As stated before, this is possible for the cell in steady growth since the metabolite concentrations do not change much through the growth cycle and the concentrations stay in the saturation region.

At this point we can use the modal matrix predictions. Over the time scale of observation, the eigenrow's weights for $M_{4}$ and $P_{4}$ appear in a constant ratio $a: 1$ in the three key modes (see Table I). Combining $P_{4}$ and $M_{4}$ as one metabolite pool, we can write the following dynamic description for this pool:

$$
\frac{d}{d t}\left(P_{4}+a M_{4}\right)=\alpha_{P_{4}} V-\gamma_{4} \frac{d M_{4}}{d t}+a \frac{d M_{4}}{d t}
$$

Since $a$ takes the value $a=1.1=\gamma_{4}$, we get

$$
\frac{d}{d t}\left(P_{4}+1.1 M_{4}\right)=\alpha_{P_{4}} V
$$

We can therefore combine $P_{4}$ and $M_{4}$ into a pool, $P_{4}+$ $1.1 M_{4}$, which describes the motion of these metabolites on the growth time constant. This correspondence between the structure of the stoichiometric matrix and the modal matrix is the heart of the natural pool formation. Similar analysis is carried out for the pools of amino acids and protein, and ribonucleotides and RNA as indicated by the modal matrix.

\section{Linear Model}

Following the general procedure indicated above, a simple three pool growth description of $E$. coli metabolism can be formulated as shown in Figure 3. This model is a direct result of the analysis of the SCM according to the aforementioned guidelines.

The dynamics of the three pools are described by:

$$
\begin{gathered}
\frac{d}{d t}\left(\mathrm{Pool}_{1}\right)=x_{1}\left(\nu_{\text {ana }}\right)(V)-\nu_{\mathrm{DNA}} \\
\frac{d}{d t}\left(\mathrm{Pool}_{2}\right)=x_{2}\left(\nu_{\text {ana }}\right)(V) \\
\frac{d}{d t}\left(\mathrm{Pool}_{3}\right)=x_{3}\left(\nu_{\text {ana }}\right)(V)
\end{gathered}
$$

with the reaction rates:

$$
\begin{gathered}
\nu_{\text {ana }}=\text { const. } \\
\nu_{\mathrm{DNA}}=\nu_{\text {md }} N_{\text {forks }} ; \quad \nu_{\text {md }}=\text { const. }
\end{gathered}
$$

The mass balance on glucose is given by:

$$
\frac{d}{d t}(\mathrm{Glu})=\nu_{\mathrm{in}}-\nu_{\mathrm{cat}}-\left(\nu_{\mathrm{ana}}\right)(V)=0
$$

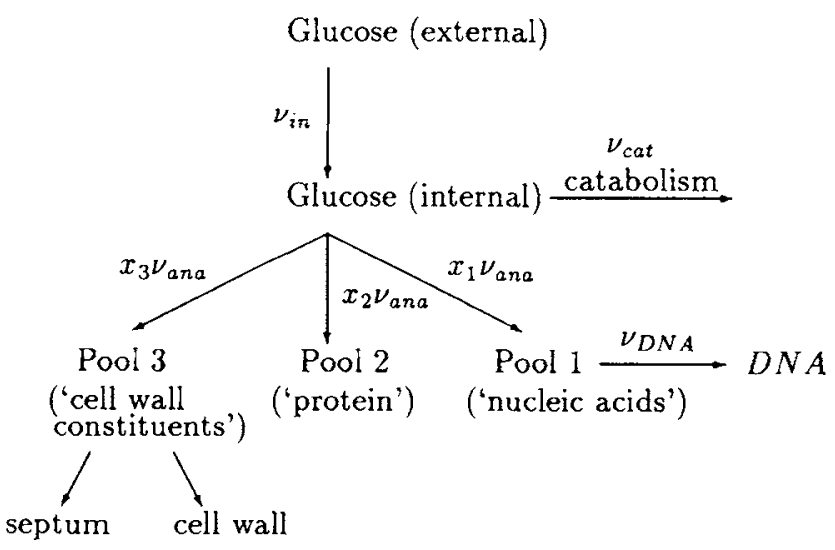

Figure 3. Reduced growth model of $E$. coli. Here $\nu_{\mathrm{in}}$ is the total substrate uptake rate, $\nu_{\mathrm{cat}}$ the catabolic rate, $\nu_{\mathrm{ana}}$ the anabolic rate and $\nu_{\mathrm{DNA}}$ the DNA synthesis rate; $x_{1}, x_{2}$, and $x_{3}$ accounts for the fractional split of anabolic flux that contributes to the formation of the macromolecular pools. 
The transient mass balance on glucose accounts for dissimilation requirements that provides ATP and the reducing power needed for biosynthesis, membrane energization and the transport reaction that drives the nutrients from external environment to the intracellular surroundings. The modal structure shows that glucose is a fast variable and hence the concentration of glucose is considered to be in a quasisteady state.

The anabolic rate $\nu_{\text {ana }}$ determines the formation of the pools and $x_{1}, x_{2}$, and $x_{3}$ accounts for the fractional split of this rate into the corresponding fluxes for the growth of these physiologically significant pools (note that $x_{1}+$ $x_{2}+x_{3}=1$ ). The rates $\nu_{\text {in }}$ and $\nu_{\text {cat }}$ are the total substrate uptake and catabolic rates and $V$ is the volume of the growing cell. The net rate of DNA synthesis is $\nu_{\mathrm{DNA}} ; \nu_{\mathrm{md}}$ is the maximum rate of the production DNA; and $N_{\text {forks }}$ is the total number of DNA forks growing in the cell.

\section{Auxiliary Decision Making Events}

As observed from the model topology, Figure 2, the dynamics of cell morphology and the control of initiation of DNA synthesis are direct functions of the amount of macromolecules present. The cell geometry and the mechanism for the control of DNA initiation are assumed to be the same as in the original model.

1) DNA synthesis. A zeroth-order synthetic rate of DNA is assumed for this simple linear model. This assumption is justified by the fact that the DNA synthesis proceeds at a constant rate; except that this constant rate is function of number of forks that propagates during the course of cell division.

2) Septum/cell wall synthesis. The enzyme molecules catalyzing the cell wall and the septum wall formation are $E_{2}$ and $E_{3}$, respectively. Their dynamics are related to the protein pool as:

$$
\begin{gathered}
\frac{d E_{2}}{d t}=e_{2} \frac{d}{d t}\left(\mathrm{Pool}_{2}\right) \\
\frac{d E_{3}}{d t}=e_{3} \mathrm{Pool}_{2}
\end{gathered}
$$

where $e_{2}$ and $e_{3}$ are kinetic constants.

3) Cell division. The septum wall is synthesized after the termination of DNA synthesis. The cell divides when the septum wall formed matches the cell width (see Fig. 2 and the "Common Attributes of SCM and the Three-Pool Model ${ }^{11 "}$ "section for details).

This linear reduced model is dynamically equivalent to the full structured model over the time scale of growth. Since this reduced description is obtained through a linear analysis, the predictions of the transient mass balances are unable to show the response of the cell to changes in the external nutrient concentrations. However, the model prediction compares well with the full SCM for a specified external glucose concentration.

\section{Nonlinear Formalism - The Complete Model}

Essential nonlinear dynamics are exhibited by the structured SCM when significant shifts in the external glucose concentrations occur. These effects are reflected by the fact that a few metabolite concentrations vary from their saturation region in a given rate law and temporarily take values that are on the order of $K_{m}$ values. The computer cells take ca. five to six generations to adjust their internal metabolite concentrations to new growth conditions. Once the cells are in a steady growth pattern they maintain a specific relationship between cell division, DNA termination and initiation of replication.

The metabolic control signals that account for shifting growth pattern in the SCM are based on glucose concentration. These few but significant hyperbolic terms are readily identified via model inspection. The mathematical form of the complete model is the same except that $\nu_{\mathrm{ana}}$ and $\nu_{\mathrm{DNA}}$ take the following form:

$$
\begin{aligned}
\nu_{\text {ana }} & =\frac{V_{\mathrm{ma}}(\mathrm{Glu} / V)}{K_{\mathrm{ma}}+(\mathrm{Glu} / V)} \\
\nu_{\mathrm{DNA}} & =\frac{V_{\mathrm{md}}(\mathrm{Glu} / V)}{K_{\mathrm{md}}+(\mathrm{Glu} / V)} N_{\text {forks }}
\end{aligned}
$$

The full model equations for the metabolic dynamics and the associated cell morphology and decision making events of the bacterial growth are given in Tables II and III. The parameter values, which are non adjustable, for the complete three-pool single cell model (TPSCM) are given in Table IV. This three pool reduced model and the full SCM show essentially identical growth patterns. In the "ThreePool Model Predictions" section, we present a comparison of the predictions of the two models of physiologically important features as the macromolecular compositions, cell morphology etc. There is an order of magnitude of reduction in the number of kinetic parameters to a significant few and that simplifies making a priori predictions of the transient behavior of cell growth.

\section{COMMON ATTRIBUTES OF SCM AND THE THREE-POOL MODEL}

The auxiliary cell machinery that couples with the metabolic growth description is envisaged to work as suggested in the literature. ${ }^{11-14}$ We have not changed this auxiliary model description primarily for two reasons:

1) the auxiliary decision making events are directly related to the predictions of the metabolic part of the model and

2) the description of cell geometry and DNA initiation are based on certain independent set of assumptions. Consequently, these attributes of the full SCM were incorporated into the reduced three-pool description of the bacterial growth with appropriate justification. 
Table II. Three-pool single-cell model equations. The equations represents dynamic mass balance on each metabolite where $V_{\mathrm{ma}}$ and $V_{\mathrm{md}}$ are the maximum anabolic and $D N A$ synthesis rate and $K_{\mathrm{ma}}$ and $K_{\text {mo }}$ are the corresponding saturation constants; $N_{\text {forks }}$ is the total number of forks in the DNA molecule; $G l u_{\text {ext }}$ represents the extracellular glucose concentration; and Glu is the intracellular glucose mass. The surface area of the cell is represented by $A ;(P / O)$ is the molecules of ATP synthesized per atom of oxygen consumed; $M_{c}$ is the maintenance energy term that includes the contribution due to membrane leakage, ion transport, and reducing power for biomass.

Metabolic Dynamics

$$
\begin{gathered}
\frac{d}{d t}\left(\mathrm{Pool}_{1}\right)=x_{1}\left(\nu_{\mathrm{ana}}\right)(V)-\nu_{\mathrm{DNA}} \\
\frac{d}{d t}\left(\mathrm{Pool}_{2}\right)=x_{2}\left(\nu_{\mathrm{ana}}\right)(V) \\
\frac{d}{d t}\left(\mathrm{PoOl}_{3}\right)=x_{3}\left(\nu_{\mathrm{ana}}\right)(V) \\
\nu_{\text {in }}-\nu_{\mathrm{cat}}-\left(\nu_{\mathrm{ana}}\right)(V)=0
\end{gathered}
$$

Rate laws

$$
\begin{gathered}
\nu_{\mathrm{ana}}=\frac{V_{\mathrm{ma}}(\mathrm{Glu} / V)}{K_{\mathrm{ma}}+(\mathrm{Glu} / V)} ; \quad V=\frac{\mathrm{Pool}_{\mathrm{I}}+\mathrm{Pool}_{2}+\mathrm{Glu}+\mathrm{DNA}}{\rho_{\mathrm{cyto}}}+\frac{\mathrm{Pool}_{3}}{\rho_{\mathrm{cw}}} \\
\nu_{\mathrm{DNA}}=\frac{V_{\mathrm{md}}(\mathrm{Glu} / V)}{K_{\mathrm{md}}+(\mathrm{Glu} / V)} N_{\mathrm{forks}} \\
\nu_{\mathrm{in}}=\nu\left(\frac{\mathrm{Glu}_{\mathrm{ext}}}{K_{G}+\mathrm{Glu}_{\mathrm{cxt}}}\right)\left(\frac{\mathrm{K}_{\mathrm{ic}}}{\mathrm{K}_{\mathrm{iG}}+(\mathrm{Glu} / \mathrm{V})^{4}}\right) A ; \quad A=f_{s} \mathrm{Pool}_{3} \\
\nu_{\mathrm{cat}}=\left(\frac{\frac{d}{d t}(\mathrm{ATP})+\frac{d}{d t}\left(M_{c}\right)(P / O)}{[4+(12-4 z)(P / O)]}\right) 180 ; \quad z=\mu_{\frac{2}{2}} \frac{\frac{d}{d t}\left(\mathrm{Pool}_{3}\right)}{\frac{d}{d t}\left(\mathrm{Pool}_{3}\right)+K_{\mathrm{z}}}
\end{gathered}
$$

Metabolic energy requirements

$$
\frac{d}{d t}(\mathrm{ATP})=\eta_{1} \frac{d}{d t}\left(\mathrm{PoOl}_{1}\right)+\eta_{2} \frac{d}{d t}\left(\mathrm{Pool}_{2}\right)+\eta_{3} \frac{d}{d t}\left(\mathrm{Pool}_{3}\right)+\eta_{d} \frac{d}{d t}(\mathrm{DNA})+\eta_{\nu} \frac{d}{d t}(V)
$$

Redox requirements

$$
\frac{d}{d t}\left(M_{e}\right)=\eta_{a} A+\eta_{m \mathrm{I}} \rho_{\text {cell }} \frac{d}{d t}(V)
$$

\section{Chemical Energy for Biosynthesis}

The principle carrier of biologically utilizable chemical energy is ATP and all the energy requiring processes in the bacterial cells are directly or indirectly coupled to the conversion of ATP to ADP and $P_{i}$. The cost of metabolic growth is obtained by adding the costs of starting biosynthetic material and the conversion cost of the coupling factors used in the biosynthesis. ${ }^{31,34,35}$ Such a calculation serves as a basis of estimating ATP equivalents required in the synthesis of the metabolite pools. Such prices of the metabolic growth are given in Table V.

The incomplete oxidation of glucose and the energy spilling mechanisms are incorporated as such from the original model. However, the requirements of ATP and the available reducing power for biosynthesis in our model are based on equivalent metabolite pools. The amount of ATP generated from substrate level phosphorylation and oxidative phosphorylation is given by; $;^{12}$

$$
\begin{aligned}
\text { ATP }= & \text { substrate level phosphorylation } \\
& + \text { oxidative phosphorylation }
\end{aligned}
$$

$$
\begin{aligned}
\operatorname{ATP}=4\left(\frac{\nu_{\text {cat }}}{180}\right) & +\left[\frac{\nu_{\text {cat }}}{180}(12-4 z)\right. \\
& - \text { (maintenance energy })] \cdot(P / O)
\end{aligned}
$$

The substrate level phosphorylation and the oxidative phosphorylation rates are related to eqs. (7) and (8) in Table II. Maintenance energy includes the contributions due to membrane leakage, ion transport, and reducing power for biomass. $(P / O)$ is the number of molecules of ATP synthesized per oxygen atom consumed and $z$ is the moles of acetate formed per mole of glucose dissimilated. The parameter values for calculation of these energy requirements are given in Table V.

\section{Growth of Cellular Geometry}

Since the reduced model also treats the cell as an expanding bioreactor, the volume of the cell and its geometrical dimensions are continuously varying with time. The cell is 
Table III. Associated cell morphology and decision making events of the cell. The constants for the cell and septum wall catalyzing enzymes are $e_{2}$ and $e_{3}$; ARP and RP are the antirepressor and repressor proteins, respectively; and $N_{0}$ is the number of origins for DNA replication. The surface area of the cell is given by $A ; V$ is the cell volume; $W$ is the cell width; $S l$ is the septum length; and $S e$ the septum wall formed. This description is directly taken from the SCM (ref. 12).

Cell wall and septum wall catalyzing enzymes

$$
\begin{gathered}
\frac{d E_{2}}{d t}=e_{2} \frac{d}{d t}\left(\mathrm{Pool}_{2}\right) \\
\frac{d E_{3}}{d t}=e_{3} \mathrm{Pool}_{2}
\end{gathered}
$$

Decision making events - ARP and RP dynamics

Cell geometry

$$
\begin{gathered}
\frac{d}{d t}(\mathrm{ARP})=k_{\mathrm{ARP}} \frac{d}{d t}\left(\mathrm{Pool}_{3}\right) \\
\mathrm{RP}=k_{\mathrm{RP}} N_{0}\left(\frac{\mathrm{k}_{\mathrm{iRP}}}{k_{\mathrm{iRP}}+\left[\left(\frac{d \mathrm{PoOl}_{2} / d t}{\mathrm{PoOl}_{2}}\right)_{\max } \frac{\mathrm{PoOl}_{2}}{V}-\left(\frac{d \mathrm{Pool}_{2} / d t}{V}\right)\right]}\right)
\end{gathered}
$$

$$
\begin{gathered}
A=\pi W^{2}+\pi W L+2 \pi W L S l \\
V=\frac{1}{6} \pi W^{3}+\frac{1}{4} W^{2} L+\frac{1}{2} \pi W^{2} S L-\frac{2}{3} \pi S l^{3} \\
S e=2 \pi W S l \\
\frac{d}{d t}(S e)=\left(\frac{E_{3}}{E_{2}+E_{3}}\right) \frac{d}{d t}(A)
\end{gathered}
$$

Table IV. Parameter values and constants for the model equations.

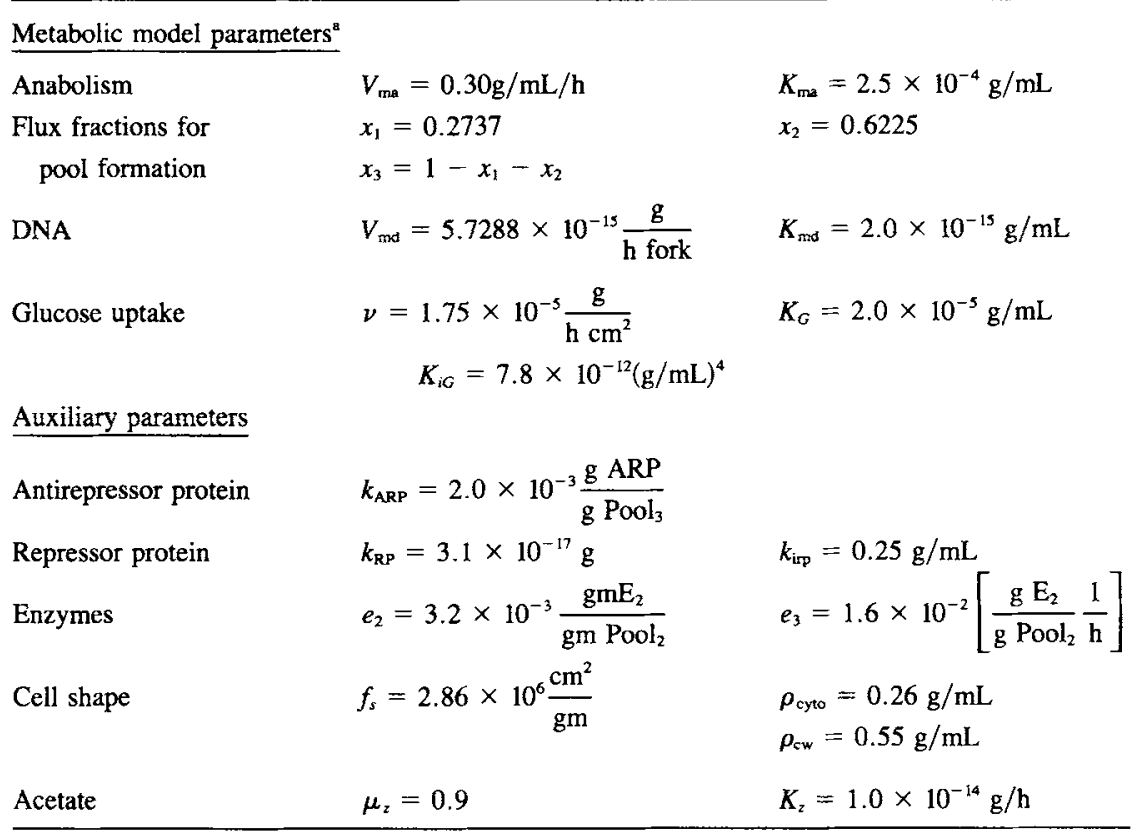

${ }^{\text {a }}$ There is an order of magnitude reduction in the number of these parameters as compared to SCM.

Table V. Prices of metabolic growth.

\begin{tabular}{ll}
\hline Pool $_{1}, \eta_{1}$ & $0.0318 \mathrm{~mol} \mathrm{ATP} / \mathrm{g}$ \\
Pool $_{2}, \eta_{2}$ & $0.0415 \mathrm{~mol} \mathrm{ATP} / \mathrm{g}$ \\
Pool $_{3}, \eta_{3}$ & $0.0097 \mathrm{~mol} \mathrm{ATP} / \mathrm{g}$ \\
DNA, $\eta_{d}$ & $0.0071 \mathrm{~mol} \mathrm{ATP} / \mathrm{g}$ \\
Unaccounted ATP, $\eta_{\nu}{ }^{\mathrm{a}}$ & $0.00633 \mathrm{~mol} / \mathrm{mL}$ \\
Membrane energization, $\eta_{a}{ }^{\mathrm{a}}$ & $9.57 \times 10^{-8} \mathrm{~mol} / \mathrm{h} \mathrm{cm}{ }^{2}$ \\
Transport of ions, $\eta_{m}{ }^{2}$ & $0.0282 \mathrm{~mol} / \mathrm{g} \mathrm{cell}$ \\
\hline
\end{tabular}

a These costs are taken from ref. 12 after proper corrections for pool sizes. 
assumed to be cylindrical in shape with closed hemispherical ends. ${ }^{11}$ All the mathematical expressions for the cell volume, the surface area, and the cross wall (septum wall) follow straight from mensuration science (see Table III). The volume of the cell is taken as the sum of the three metabolite pools divided by their corresponding density. The surface area of the cell is expressed as the amount of cell area formed per mass of the cell envelope pool. The septum wall, which is synthesized after the DNA termination, is taken as a certain fraction of the cell envelope synthesized. This fraction is determined by the amount of enzyme molecules $E_{2}$ and $E_{3}$ formed. These enzyme molecules catalyzing the septum and cell wall formation are expressed as a function of the protein pool (eq. (18) and (19)).

\section{Mechanism for DNA Initiation}

Manifestation of numerous mechanisms in the literature $^{36-38}$ for the control of DNA initiation shows that the exact hypothesis for such a formulation is difficult to determine. The SCM uses the observation of Fralick and Messer and colleagues ${ }^{27-29}$. Since our primary effort is to replicate the full SCM with a minimal complexity in the form of postulated three pool model, we use the same formulation for DNA synthesis (see Table III). Again the difference is that our model assumes dependency of this mechanism on simple macromolecule pools rather than the precursormacromolecule relationship of the original model. The DNA synthesis is initiated when the antirepressor protein (ARP), which is continuously formed as a certain fraction of the cell envelope, exceeds the repressor protein (RP) made at DNA initiation. Like the SCM, our model assumes the continuous formation of ARP as a certain fraction of the cell envelope pool and the production of RP as a burst that is modulated by the protein pool synthesis rate.

\section{THREE-POOL MODEL PREDICTIONS AND ITS COMPARISON TO SCM AND EXPERIMENTS}

The aforementioned analysis and the development of the three pool model is aimed at answering two important questions about bacterial growth:

1) What determines the growth rate in a given medium?

2) How does the cell regulate its growth under a variety of growth conditions and what are the important dynamic variables and parameters that regulate cellular growth?

One of the obvious checks before any attempts are made to answer these questions is to first compare the quality of the three-pool model predictions with the full SCM. To test the validity of our model, several computer experiments were carried out using the parameters given in Table III. The parameters obtained to predict the cell's growth behavior are strictly according to the model reduction technique as described earlier. In the model reduction procedure, no adjustments of parameters are made to obtain a fit to the data and known predictions.

\section{Shifts between Growth Conditions}

Simulated response to nutritional changes can be carried out by changing the limiting substrate concentration (in our case glucose) of the external environment. The metabolic capabilities of the bacterium in response to such a shift is strong, and they are reflected in the quick adaptability of the bacterium to a new growth surrounding in only a few generations. The validity of the three-pool model is confirmed by performing such downshift and upshift experiments.

\section{Response of the Model to Downshift in External Glucose Concentration}

The reduced TPSCM was allowed to run for several generations for a 45 minute cell. Then the abiotic environment was suddenly decreased in a step down fashion from external glucose concentration ${ }_{A}$ to concentration ${ }_{B}$ and the response of the model was observed over a number of generations. The cell takes five to six generations to achieve a new steady-state growth rate (Fig. 4). This is in excellent comparison with the SCM predictions. A steady decrease in the growth was observed unlike the SCM which predicts a very long second generation of growth.

\section{Response of the Model to Upshift in External Glucose Concentration}

The computer simulations were performed again by stepping up the external glucose concentration from concentration $_{B}$ to concentration ${ }_{A}$. A steady increase in the growth rate was observed and the cell's response was spread over a few generations to come back to its steady state growth rate (Fig. 4).

Such predictions of the cell's growth rate under varying growth conditions are in accord with the full SCM and the

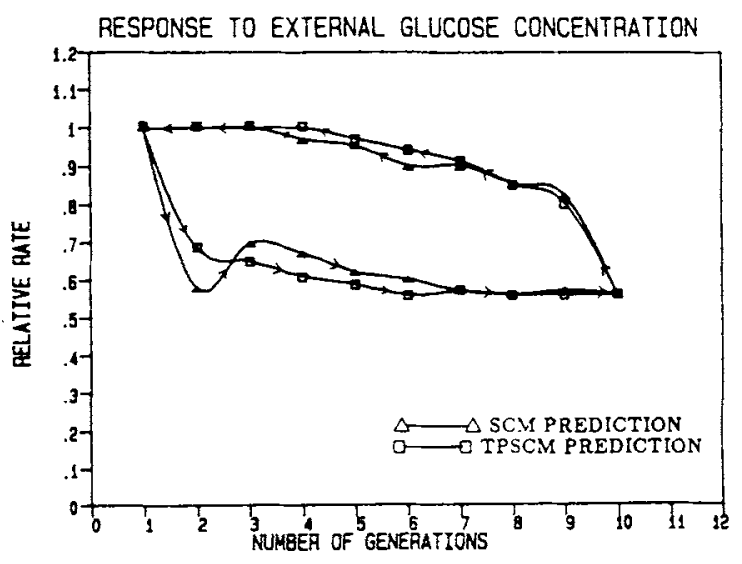

Figure 4. Response of the model to changes in external glucose concentration. The external glucose concentration is stepped down from $1 \times 10^{-3}$ to $4.4 \times 10^{-6} \mathrm{~g} / \mathrm{L}$. For stepup, the glucose concentration is changed from $4.4 \times 10^{-6}$ to $1 \times 10^{-3} \mathrm{~g} / \mathrm{L}$. The computer cell takes a few generations to adjust to its new steady growth: $(\triangle \triangle \triangle)$ SCM predictions and $(\square-\square)$ TPSCM predictions. 
reported experimental trend in the literature. ${ }^{31,39}$ Furthermore, it reflects the inherent plasticity of the TPSCM to respond to such a shift and exhibit the non-linear behavioral characteristics of the cell.

\section{Growth Rate Dependence on Substrate Concentration}

Figure 5 shows the superimposed plots of the TPSCM model predictions to experimental data and those of the full SCM. Overall, the predicted TPSCM response tracks the experimental data and is, in particular, consistent with the response curve of the full SCM. The model's projected trend is in close agreement with the available experimental data on the growth rate. ${ }^{13}$ With only a few kinetic parameters in the reduced model, the sensitivity of the overall growth mechanism to these parameters can be readily studied.

Furthermore, an order of magnitude estimation for the specific growth rate can be made simply based on the initial conditions specified for the cell. This is achieved by calculating the modal matrix and the hierarchy of the relaxation times. The unstable growth mode and the negative time constant predicts the approximate doubling time for the cell. The relation in eq. (9) is used to calculate the specific growth rate. Figure 6 show that the order of magnitude estimation of the doubling time and the growth rate obtained by complete simulation are in good agreement.

\section{Macromolecule Composition Profile during the Division Cycle}

Figure 7 shows the reduced model predictions for the macromolecule composition profiles through the cell cycle for the 45-min cell. The time axis is scaled to the total cycle time and the macromolecule compositions (pool size) are scaled to their initial value. It is observed that the pools grow exponentially with time and at the end of the division cycle,

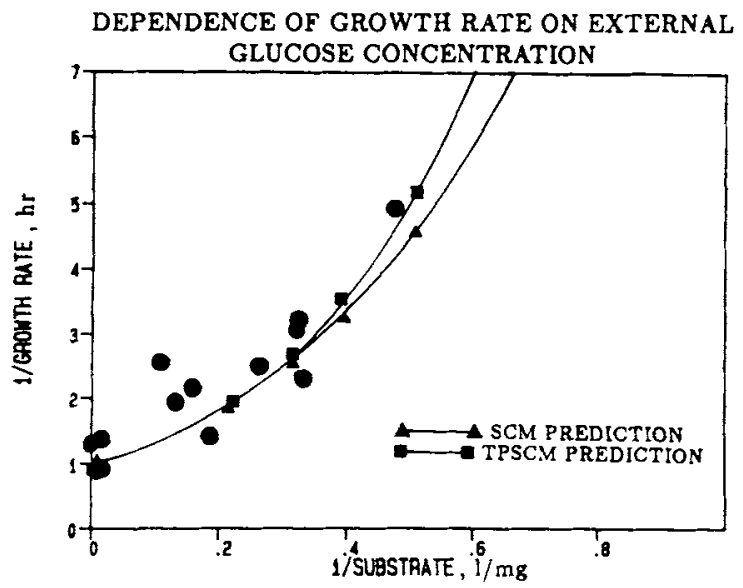

Figure 5. Dependence of growth rate on external glucose concentration: (O) experimental data (ref. 12), ( $\_-\perp$ ) the SCM model predictions, (I- - ) reduced three pool model predictions. The growth dependence of external glucose is a natural response of the model and is not fitted to the data.

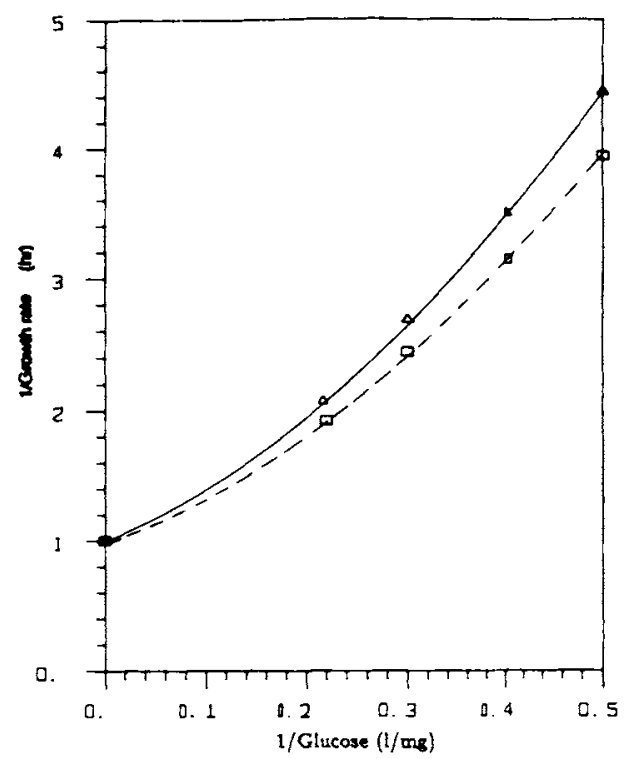

Figure 6. Predicted doubling time as a function of external glucose concentration: $(\triangle)$ predicted time from unstable mode, and $(\square)$ simulated time. The predicted doubling time is calculated from the time constant predicted by the unstable mode and by using eq. (6) in the text. No full integration of the model equations is involved.

the pool composition is doubled as expected. Similar observations are made for cells with other cycle times.

\section{Cell Morphology}

The reduced TPSCM of $E$. coli has the ability to predict the cell morphology over a variety of growth conditions for glucose limited growth. If the model's prediction of the macromolecule pool size is sufficiently accurate, the prediction of cell geometry is a natural consequence of the model. A representative plot, Figure 8 , shows the variation of cell width during the division cycle. The model predicts that the cell width does vary during the division cycle ${ }^{40}$ and the maximum deviation from the average width occurs when there is observable septum wall formation. No noticeable increase in the cell width is observed in the very beginning of the growth phase as predicted by the full SCM. However, the largest deviation of the cell width is observed for the slowest growing cells (with large doubling times) and this is in accord with the full model and the findings of Truebe and Woldringh. ${ }^{40}$

\section{Cell Composition}

About $95 \%$ of the dry weight of $E$. coli and other microorganisms consists of macromolecules. A typical analysis reflects that protein account s for approximately $52 \%$ and nucleic acids for $19 \%$ of the cell's dry mass. The cell envelope constituents accounts for $17-18 \%$ of the cell. Protein, RNA, and DNA together account for ca. $80 \%$ of the dry weight of the cell. Figure 9 show the model prediction of how the relative amounts of these macromolecules vary with the growth rate. Jacobsen ${ }^{41}$ reported the dependence of 


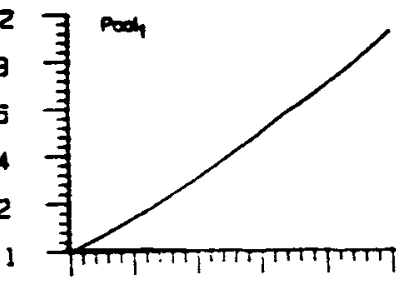

0.0 .20 .40 .60 .81

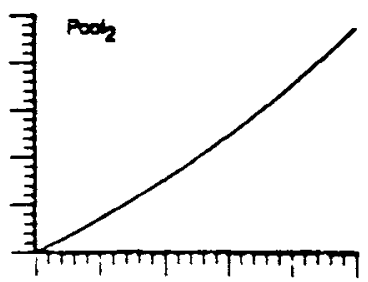

0.0 .20 .40 .50 .81

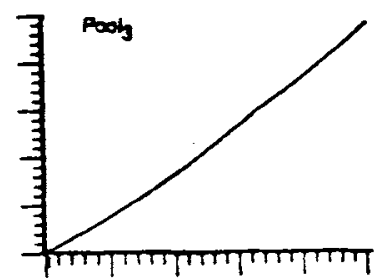

0.0 .20 .40 .60 .81

Figure 7. Relative macromolecular composition profile for the cycle time of $45 \mathrm{~min}$ cell. The $x$-axis is scaled to the total doubling time and the $y$-axis is scaled to the pools' composition of freshly born cell.

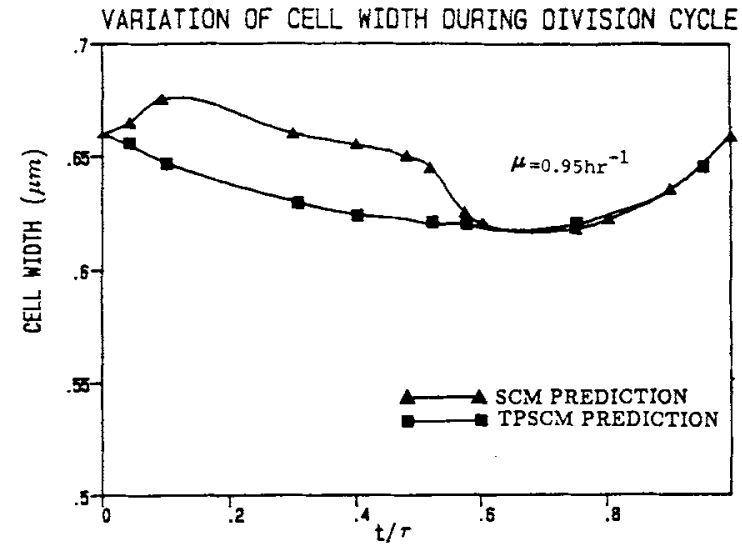

Figure 8. Variation of cell width during the division cycle of the cell for $0.95 \mathrm{~h}^{-1}$. The cell width slowly decreases and then steadily increases with the growth: (4) SCM prediction and (๘) TPSCM prediction.

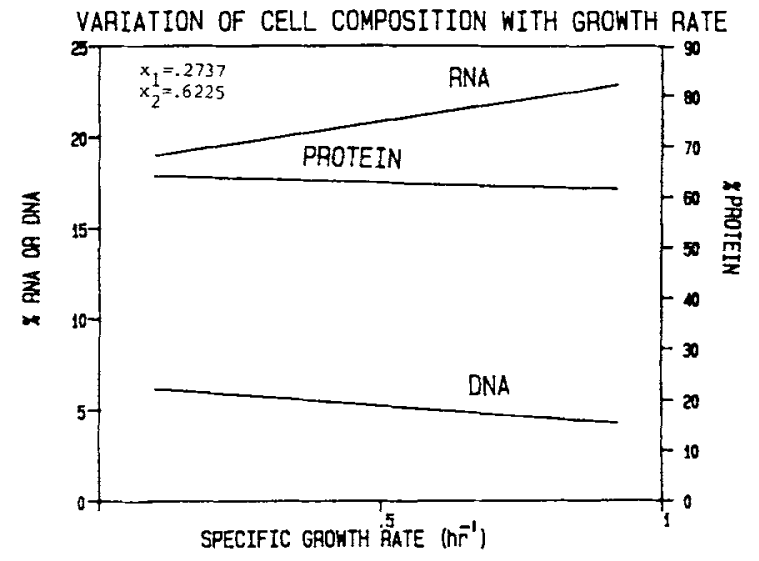

Figure 9. Variation of macromolecular composition with the growth rate These prediction are for the $E$. coli cells growing in a chemostat with glucose as a limiting nutrient; $x_{1}$ and $x_{2}$ are the fractions of anabolic flux that accounts for the growth of physiologically significant macromolecular pools.

protein, RNA and DNA content on the growth rate in a glucose-limited chemostat. The projected trend is in accord with this work. Both protein and DNA content fall with the increasing growth rate where as total RNA content increases with the increasing growth rate.

\section{Cell Energy Budget}

One of the effective test $s$ of energy accounting in the cell is the determination of growth yield. The formation of new material (net increase in cell mass) in the cell primarily depends upon the available energy for anabolism and the energy generated during catabolism. Growth yields, $Y_{\text {glu }}$, are commonly expressed as grams of dry weight of cell per grams of substrate consumed (glucose is the substrate). An equation describing the relation between the growth yield and the specific growth rate has been derived by $\mathrm{Pirt}^{42}$;

$$
\frac{1}{Y_{\text {glu }}}=\frac{1}{Y_{\text {glu }}^{\max }}+\frac{m_{\mathrm{glu}}}{\mu}
$$

This relation expresses the fact that during the organism's growth, the consumption of substrate is divided between growth dependent and growth independent processes.

Figure 10 shows a linear regression of the TPSCM predictions; which gives $m_{\mathrm{glu}}=0.25$ and $Y_{\mathrm{glu}}^{\max }=0.47$. These values are very well in accord with SCM prediction of $0.21 \mathrm{~g}$ glucose $/ \mathrm{g}$ cell $\mathrm{h}$ and $0.49 \mathrm{~g}$ cell $/ \mathrm{g}$ glucose consumed and the reported yield data from Seniors. ${ }^{43}$

\section{DISCUSSION}

Biochemical regulation of biological processes are generally characterized by large dimensionality, strong interaction among the system variables and nonlinearity. It is

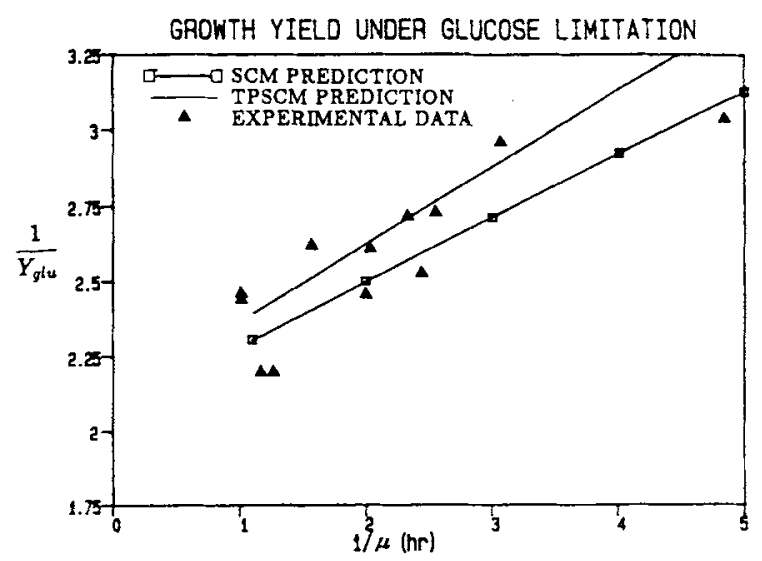

Figure 10. Double reciprocal plot of growth yield and specific growth rate. The SCM and TPSCM predictions are compared to the data for $E$. coli B/r-A (ref. 12). The solid line represents the TPSCM predictions: $(\square-\square)$ represents SCM predictions. Parameter $Y_{g \mathrm{gu}}^{\max }$ obtained from TPSCM is 0.47 as compared to 0.48 of SCM. Parameter $m_{\mathrm{glu}}$ for TPSCM is 0.251 as compared to 0.20 from SCM. 
easy to realize that the successful application of a mathematical model of such complex system for the design, operation, and optimization of a bioreactor greatly depends upon the mathematical tractability and accurate description of process reality. Furthermore, the model should contain a few significant parameters which can be either measured independently or estimated by an objective set of criteria. The TPSCM is simple enough for accurate predictions of $E$. coli growth and it provides an opportunity for its employment to study reactor scaleup and reactor configuration strategies to make optimum use of this microorganism.

In this article, a biochemically based kinetic model of $E$. coli under the glucose limiting condition of growth has been examined and taken as a starting point for the development of a tractable but physiologically realistic growth model. The structure of SCM was analyzed using temporal decomposition and a study of relaxation time hierarchy. The reduced three-pool single cell model and its structure has been determined based entirely on the information provided by the modal matrix and the inherent dynamic characteristics of the SCM. The model parameters postulated in the reduced model are derived from the parameters of the full SCM. TPSCM successfully and simultaneously predicts the macromolecular composition, cell size and shape, DNA initiation and termination points, and the growth rate and yield of the cell under glucose limitation. TPSCM predictions are essentially the same as those of the detailed SCM.

Temporal decomposition results in an elegant dynamic interpretation of the biochemically based structured $E$. coli model. The interaction between the low-molecular-weight precursors, $P_{i}$, and the macromolecules, $M_{i}$, lead to the formation of physiologically significant metabolic pools that are comprised of the macromolecules and their precursors. The quality and extent of the TPSCM predictions should not come as a surprise; the model accounts for only the important transients required for the description of growth and has been stripped of all unnecessary metabolic description.

Since the model explicitly accounts for the three important macromolecular pools, necessary details can be added to description of each of the pools depending on the particular application of the model, e.g., significant refinement can be made into the pool of nucleic acids to account for the plasmid replications. The model can be extended to describe cellular growth under nitrogen source limitations. Furthermore, because of the simplicity of the model, scaling techniques can be applied to this reduced model to extract essential dimensionless groups and property ratios that characterize growth dynamics; and the TPSCM therefore has the potential to become a generic bacterial growth model.

The authors thank Professor M. L. Shuler for the critical reading of this manuscript and for his many valuable comments.

\section{References}

1. J. D. Watson, Molecular Biology of the Gene (Benjamin, Menlo Park, CA, 1975).

2. E. Pollard, Am. Naturalist, 94, 71 (1960).

3. W. G. Yeisley and E. C. Pollard, J. Theor. Biol., 7, 485 (1964).

4. F. Heinmets, J. Theor. Biol., 6, 60 (1964).

5. R. Weinberg and B. P. Zeigler, "Computer Simulation of a Living cell: Multilevel control systems," Report 08228-17-T, University of Michigan, Ann Arbor, MI, 1969.

6. E. J. Davison, Bull. Math. Biol., 37, 427 (1975).

7. A. Harder and J. A. Roels, Adv. Biochem. Eng., 21, 55 (1982).

8. F. Chiam and I. J. Harris, Biotechnol. Bioeng., 24, 37 (1982).

9. D. Ramkrishna, A. G. Frederickson, and H. M. Tsuchiya, Biotechnol. Bioeng., 9, 129 (1967).

10. D. Ramkrishna, ACS Symp. Ser., 207, 162 (1983).

11. M. L. Shuler, S. Leung, and C. C. Dick, Ann. NY Acad. Sci., 326, 35 (1979).

12. M. M. Domach, S. K. Leung, R.E. Cahn, G. G. Cocks, and M.M. Shuler, Biotechnol. Bioeng., 26, 204 (1984).

13. M. M. Domach, "Refinement and Use of a Structured Model of a Single Cell of Escherichia coli for the Description of Ammonialimited Growth and Asynchronous Population Dynamics," Ph.D. thesis, Cornell University, Ithaca, NY, 1983.

14. M. M. Domach and M.L. Shuler, Biotechnol. Bioeng., 26, 877 (1984).

15. M. M. Ataai and M. L. Shuler, Biotechnol. Bioeng., 27, 1027 (1985).

16. S. B. Lee and J.E. Bailey, Biotechnol. Bioeng., 26, 66 (1984).

17. S. B. Lee and J. E. Bailey, Plasmid, 11, 151 (1984)

18. S. B. Lee and J. E. Bailey, Plasmid, 11, 166 (1984).

19. B. O. Palsson, A. Joshi, and S. S. Ozturk, Federation Proceedings, 46, 2485 (1987)

20. B. O. Palsson, in Electrochemical activation, metabolism and perfusion of the Heart-Simulation and experimental models, S. Sideman and B. Bexar, Eds., (Martinus Nijhoff, Wordrecht, p. 584, 1987.).

21. B. O. Palsson, Chem. Eng. Sci., 42, 447, (1987).

22. B. O. Palsson, "Mathematical Modeling of Dynamics and Control in Metabolic Networks," Ph.D. thesis, University of Wisconsin, Madison, WI, 1984.

23. B. O. Palsson and E. N. Lightfoot, J. Theor. Biol., 111, 272 (1984).

24. J. G. Reich and E. E. Sel'kov, FEBS Lett., 40, S119 (1974).

25. J. G. Reich and E. E. Sel'kov, Biosystems, 7, 39 (1975).

26. J. A. Roels, Energetics and Kinetics in Biotechnology (Elsvier Biomedical Press, Amsterdam, 1983).

27. J. A. Fralick, in DNA Synthesis-Present and Future, I. Molineux and M. Kohiyama, Eds. (Plenum, New York, 1978).

28. W. Messer, L. Dankworth, R. Tippe-Schindler, J. E. Womack, and G. Zahn, in DNA Synthesis and Its Regulation, M. Goulian and P. Hanawalt, Eds. (Benjamin, Menlo Park, CA, 1975)

29. T. E. Womack and W. Messer, in DNA synthesis-Present and Future, I. Molineux and M. Kohiyama, Eds., (Plenum, New York, 1978).

30. A. C. Hindmarsh, and G.D. Byrne, "EPISODE - An experimental package for the integration of ordinary differential equations," UCID30112, Lawrence Livermore Laboratories, Livermore, CA, May 1975.

31. I. L. Ingraham, O. Maaloe, and F. C. Neidhart, "Growth of the Bacterial Cell," Sinauer Associates, Sunderland, MA, 1983.

32. I. Smith and T. Brian, EISPACK . . Matrix Eigensystem routines, G. Goos, and J. Hartmanis, Eds. (Springer-Verlag, 1976).

33. B. O. Palsson, and A. Joshi, Biotechnol. Bioeng., 29, 789 (1987).

34. D. E. Atkinson, Energy Metabolism and its Regulation (Academic, New York, 1977).

35. A. H. Stouthamer, Microbial Chemistry, J. R. Quayle, Ed. (University Park Press, Baltimore, 1979) 
36. L. Huzyk and D. J. Clark, J. Bacteriol., 108, 74 (1971).

37. C. Helmstetter, J. Mol. Biol., 84, 21 (1974).

38. R. Pritchard, P. T. Barth, and J. Collins, in Microbial Growth, 19th Symposium of the Society General Microbiology (Cambridge University Press, Cambridge, England, 1969).

39. H. Brunschede, T. L. Dove, and H. Bremer, J. Bacteriol., 142, 869 (1980).
40. F. J. Treuba and C. L. Woldringh, J. Bacteriol., 91, 2388 (1966).

41. H. Jacobsen, Ph.D. thesis, Copenhagen University, Copenhagen, 1979.

42. S. J. Pirt, Principles of microbe and cell cultivation (Wiley, New York, 1975).

43. P. J. Senior, J. Bacteriol., 123, 407 (1975). 